\title{
Chromatin-remodeling factor, RSF1, controls p53-mediated transcription in apoptosis upon DNA strand breaks
}

\author{
Sunwoo Min ${ }^{1,2}$, Keeeun Kim ${ }^{1,3}$, Seong-Gwang Kim ${ }^{1,2,3}$, Hyeseong Cho $\mathbb{B}^{1,2,3,4}$ and Youngsoo Lee $\mathbb{B}^{1,3,4}$
}

\begin{abstract}
Remodeling and spacing factor 1 (RSF1), which is one of chromatin-remodeling factors, has been linked to the DNA damage response (DDR) and DNA repair. However, the biological consequence of RSF1 deficiency in DDR in vivo and its molecular mechanisms remain unknown. Because defective DDR is related to neuropathological phenotypes, we developed neural-specific Rsf1 knockout mice. Rsf1 deficiency did not result in any neuropathological abnormalities, but prevented neural apoptosis triggered by excessive DNA strand breaks during neurogenesis. Likewise, cell death was significantly reduced in RSF1 deficient human cell lines after DNA damage, and the global transcriptome of these cells revealed that the expressions of p53 downstream genes were significantly reduced upon DNA strand breaks. Inactivation of these genes resulted from decreased binding of p53/p300 complex and subsequent reduction of H3 acetylation at their promoters. Our data show that RSF1 is necessary for p53-dependent gene expression in response to DNA strand breaks via controlling the accessibility of p53/p300 complex to its target genes and contributes to the maintenance of cellular integrity.
\end{abstract}

\section{Introduction}

Genomic stability is fundamental for proper development and lifelong functioning, and DNA repair pathways are crucial to counteract the various endogenous and exogenous sources of DNA damage that continuously challenge this stability. Numerous diseases are caused by genomic instability, including developmental defects and cancer ${ }^{1}$. As the chromatin structure in eukaryotes is a barrier to DNA damage detection and repair, chromatinremodeling complexes are important for ensuring an appropriate DNA damage response (DDR) and repair, and eventually for maintaining genomic stability ${ }^{2}$.

Correspondence: Youngsoo Lee (ysoolee@ajou.ac.kr)

${ }^{1}$ Genomic Instability Research Center, Ajou University School of Medicine, Suwon 16499, Korea

2Department of Biochemistry \& Molecular Biology, Ajou University School of Medicine, Suwon 16499, Korea

Full list of author information is available at the end of the article.

These authors contributed equally: Sunwoo Min, Keeeun Kim

Edited by M. Malewicz
Remodeling and spacing factor 1 (RSF1) is a chromatinremodeling factor initially identified as a transcriptional activator with the FACT (facilitates chromatin transcription) complex. RSF1 with SNF2h ATPase forms RSF complex in the ISWI family, which can reposition the nucleosome for transcriptional regulation ${ }^{3,4}$. Several reports demonstrated that RSF1 is overexpressed in multiple types of tumors and correlates with their aggressiveness in terms of tumor size and TNM (classification of malignant tumors) stages ${ }^{5-13}$. RSF1 overexpression was also recently correlated with pathological type, tumor aggressiveness, and TP53 status in breast cancer, such that the overall survival was shorter for breast cancer patients with tumors overexpressing RSF1 and harboring TP53 mutations ${ }^{14}$.

RSF1 may also protect against DNA damage, possibly by influencing ATM-dependent checkpoint signaling. We and other groups reported previously that RSF1 is required for proper phosphorylated H2AX $(\gamma-\mathrm{H} 2 \mathrm{AX})$ propagation and DNA damage repair ${ }^{15-17}$. Also ATM 
likely binds to and modifies RSF1 upon DNA damage ${ }^{15,17}$. ATM kinase helps maintain genomic stability in response to DNA strand breaks, and ATM mutations cause ataxia telangiectasia in human, which manifests clinically as defects of the immune system, increased tumor incidence, ataxia, and neurodegeneration ${ }^{18}$. A well-known substrate of ATM kinase is p53, which binds to the promoters of target genes and regulates target gene expressions that govern cell-cycle arrest and apoptosis in response to DNA damage $^{19}$. Gene mutations or inactivation of p53 and defects in its downstream network lead to genomic instability and promote cancer development ${ }^{20,21}$.

Although RSF1 has been implicated in the DDR, the biological consequences of RSF1 deficiency in proliferating cells under DNA damage and the molecular mechanisms for cell fate decisions remain unclear. Here, we report that RSF1 is crucial for p53-dependent cell fate determination upon DNA damage. To study the role of RSF1 in vivo, a mouse model with conditional knockout (cKO) of Rsf1 during neurogenesis was generated. Although Rsf1 was dispensable for brain development and function, the developing nervous system in Rsf 1 cKO mice was protected from cell death triggered by exogenously induced DNA strand breaks. This protection was confirmed in vitro by analyzing human RSF1 deficient cell lines. Gene-expression profiling of RNA sequencing (RNAseq) from RSF1 KO cells revealed reduced expression of p53-target genes, particularly those related to apoptosis. This reduction resulted in part from the disruption of p53/p300 binding and the consequent reduction of $\mathrm{H} 3$ acetylation on the promoters of p53-target genes upon DNA strand breaks. These data demonstrate that RSF1 is necessary for proper p53-dependent signaling induced by DNA strand breaks.

\section{Results}

\section{Rsf1 is dispensable for brain development}

Human RSF1 is immediately recruited to sites of DNA damage ${ }^{15}$, and whether this is also the case with the murine RSF1 was first examined. The kinetics of murine RSF1 accumulation to the sites of DNA strand breaks induced by microirradiation was similar to that of the human RSF1 protein (Figure S1a). To study the in vivo function of RSF1, an Rsf1 cKO animal model was generated using Flp-FRT and Cre-LoxP recombination (Figure S1b). We obtained a floxed animal model provided by the Canadian Mouse Repository at the Hospital for Sick Children, and then cross-bred with the mouse model expressing $\mathrm{Cre}$ recombinase under the control of the Nestin promoter (Nes-Cre line) to inactivate Rsf1 selectively in the neuroprogenitor cells during development. The nervous system is a good target organ to examine DDR, since the distinct developmental stage allows us to look into difference of DDR and damage repair in proliferation, differentiation, and maturation of cells, as defective DDR and damage repair in the nervous system has severe consequences in humans ${ }^{1,22}$. Furthermore, dynamic changes of chromatin environment and epigenetic regulation are one of critical regulatory factors for the proper neurogenesis ${ }^{23,24}$. In the mouse model, exon 4 of Rsf 1 encoding a part of the WHIM domains was floxed (Figure S1b, right), and then successfully deleted in a NesCre line (Rsf $1^{\text {Nes-Cre }}$ as indicated in figures) (Figure S1c), with no RSF1 protein detected throughout the central nervous system (Figure S1d).

Rsf1 cKO mice were born at an expected ratio and had a lifespan comparable to those of control (Ctrl) animals (Figure S1e). Also the cKO animals did not show any noticeable neurological phenotypes. The overall neuroanatomical features at the microscopic level were normal, with mature neurons and astrocytes observed in the hippocampus of the Rsf1 cKO brain (Fig. 1a). The distributions and densities of oligodendrocytes and interneurons as exemplified by parvalbumin positive neurons in the Rsf1 cKO cerebral cortex were comparable to those in Ctrl groups (Fig. 1b). The architecture of cerebella in Rsf1 cKO mice was also neuroanatomically intact (Fig. 1c) and these animals did not show any sign of ataxia. The protein levels of several markers for neurons, astrocytes, oligodendrocytes, and interneurons were similar between the Rsf1 Ctrl and cKO brains (Fig. 1d). Thus, we concluded Rsf1 is not essential for neurodevelopment. This result is in stark contrast to the $S n f 2 h$ cKO animal model which showed several neurological defects including cerebellar abnormalities and ataxia ${ }^{25}$. In this animal model, the $\operatorname{Snf} 2 h$ gene was also selectively inactivated in the neuroprogenitor cells ${ }^{25}$.

\section{Rsf1 is involved in apoptosis in response to DNA damage during neurodevelopment}

We also looked into embryonic brain during neurogenesis, since it has been reported that RSF1 plays a role in mitosis in vitro ${ }^{26}$. The distributions of proliferation (phosphorylated histone 3 for metaphase and proliferating cell nuclear antigen (PCNA)) and neuronal maturation (Tubulin BIII) markers were similar in the Rsf1 Ctrl and cKO embryonic brains (Fig. 2a, b). There was no ectopic expression of proliferating markers in the Rsf1 cKO embryonic brains. Also, the number of proliferating cells and the protein levels of proliferation markers in the embryonic forebrains did not differ in Rsf1 Ctrl and cKO animals (Fig. 2c, d). The normal index of proliferation and differentiation in Rsf1 cKO embryonic brains is most likely due to the fast cell cycle and characteristic symmetric/asymmetric cell division during neurogenesis ${ }^{27}$.

As RSF1 was shown to be a regulatory factor for the $\mathrm{DDR}^{15}$, we assessed the effect of Rsf1 deficiency on the repair capacity for endogenously induced DNA damage 
during neurogenesis. Endogenous DNA damage, particular strand breaks were evidently visible, only when proteins for DDR or DNA damage repair were inactivated in the developing nervous system, implying that endogenous DNA breaks naturally occur and are repaired during brain development ${ }^{22,28}$. DNA damage, visualized as foci of $\gamma-\mathrm{H} 2 \mathrm{AX}$ immunoreactivity in the nucleus, was absent from the embryonic brains of the Rsf $1 \mathrm{Ctrl}$ and cKO mice (Fig. 2a), suggesting that Rsf1 deficiency does not affect the normal repair machinery against endogenously induced DNA damage during neurogenesis.

Next, to examine the any role of Rsf1 in the DDR during neurogenesis, DNA strand breaks were exogenously introduced by administering either etoposide or hydroxyurea $(\mathrm{HU})$ to the embryos. Both reagents induced massive amounts of apoptosis, detected by TUNEL, in the developing brain, especially in the ventricular zone where neuroprogenitors reside in (Fig. 2e, f). While Rsf1 deficiency did not influence apoptosis caused by HU treatment, it was protective against apoptosis triggered by etoposide-induced DNA strand breaks (Fig. 2e, f), implying that Rsf 1 is one of key defenders against a certain type of DNA damage, particularly double-strand breaks, as suggested previously ${ }^{15}$. DNA strand breaks detected by $\gamma-\mathrm{H} 2 \mathrm{AX}$ immunoreactivity were substantially high, yet similar between Rsf1 Ctrl and cKO embryos at $2 \mathrm{~h}$ time point post etoposide treatment (Fig. $2 \mathrm{~g}$, h). This observation indicated that the etoposide treatment produced excessive DNA damage which likely surpassed the repair capability, resulting in apoptosis (Fig. 2e). However, some damaged cells in Ctrl embryonic brains were able to escape apoptosis and likely repaired DNA damage to a certain degree, as shown in reduced $\gamma-\mathrm{H} 2 \mathrm{AX}$ foci, $12 \mathrm{~h}$ posttreatment (Fig. $2 \mathrm{~h}$ ). In contrast, significant numbers of $\gamma$-H2AX foci were still remained in Rsf1 cKO embryonic brains at the same time point (Fig. 2h). These results suggest that $R s f 1$ deficiency in the developing brain hinders both DNA damage repair and apoptosis triggered by etoposide insult.

\section{RSF1 KO impairs p53 signaling and p53-induced cell death}

To study the mechanism by which RSF1 suppresses apoptosis further, transcription activator-like effector nucleases technology was used to generate RSF1 KO in a human U2OS cell line. The subG ${ }_{1}$ population, which represents dying cells, was stained with propidium iodide (PI) and quantified by fluorescence-activated cell sorting (FACS) after treatment with etoposide for $24 \mathrm{~h}$ (Fig. 3a). The induction of cell death was significantly reduced in RSF1 KO cells, comparable with in vivo results (Fig. 2e, f). Furthermore, to monitor the kinetics of apoptosis, the numbers of dead cells stained with IncuCyte Cytotox Red reagent were quantified in the images of these cells taken every $3 \mathrm{~h}$. Compared with wild-type (WT) Ctrl U2OS cells, etoposide-treated RSF1 KO cells had less red fluorescence after $24 \mathrm{~h}$ post etoposide treatment (Fig. 3b and Figure S2a), implying reduction in apoptosis after DNA strand breaks. Next, RNAseq was performed on RSF1 KO and WT Ctrl cells with and without $12 \mathrm{~h}$ etoposide treatment to investigate differential gene expression after DNA strand breaks (Figure S2b). Similar to a previous report ${ }^{29}$, the majority of transcripts were upregulated under the condition of DNA damage. An ingenuity pathway analysis (IPA) revealed that the most affected genes with differential expression between RSF1 $\mathrm{KO}$ and WT Ctrl cells are involved in the p53 signaling pathway (Fig. 3c and Figure S2c). We carried out several experiments to validate the results from bioinformatics analysis. The quantitative real-time PCR to measure the mRNA levels showed significant reduction of p53-target gene expressions, including CDKN1A (p21), BTG2 (BTG antiproliferation factor 2), $B A X$ (BCL2-associated $X)$, and BBC3 (BCL2-binding component 3, PUMA), in RSF1 deficient cells compared to RSF1 WT Ctrl cells $12 \mathrm{~h}$ after etoposide treatment (Fig. 3d). p53 dependency of these target gene expressions after DNA damage was verified using HCT116 $p 53^{+/+}$and $p 53^{-/-}$cell lines, which no or minor induction of p53-target genes was evident in $p 53$ deficient cells after DNA damage regardless the status of RSF1 (Fig. 3e, and figure S2e, S2f). This aberrant transcriptional response was not a reflection of altered p53 levels, which were maintained in RSF1 KO cells after DNA damage and were comparable to those in WT Ctrl cells (Figure S2d and S2e). We also measured the changes of p53-target proteins in the E15.5 embryonic brains after etoposide treatment. p53 stabilization was similar in Rsf1 WT and cKO embryonic brains after etoposide treatment, yet p53-target proteins including BAX, PUMA, NOXA, and p21 were less induced in Rsf1 cKO embryonic brain compared to those of Rsf1 WT brains after the drug treatment (Fig. 3f) similar to the in vitro analysis (Fig. 3d and Figure S2d). These data suggest that there is a connection between RSF1 deficiency and the gene expression of p53-target genes upon DNA strand breaks.

\section{RSF1-dependent regulation of p53 transcriptional activity upon DNA damage}

In response to DNA damage, p53 activity regulates the p53-mediated transcriptional response of its target genes. To test whether RSF1 regulates p53 activity, first we examined the activity of p53 upon DNA damage in RSF1 WT Ctrl and KO cells. Luciferase activity with p53 binding sites (PG13) was significantly increased in RSF1 WT Ctrl after etoposide treatment, yet the activity remained unchanged in RSF1 $\mathrm{KO}$ in the same condition (Fig. 4a). The negative control with the binding site mutants (MG15) did not show any significance in luciferase activity (Fig. 4a). This result suggests that RSF1 

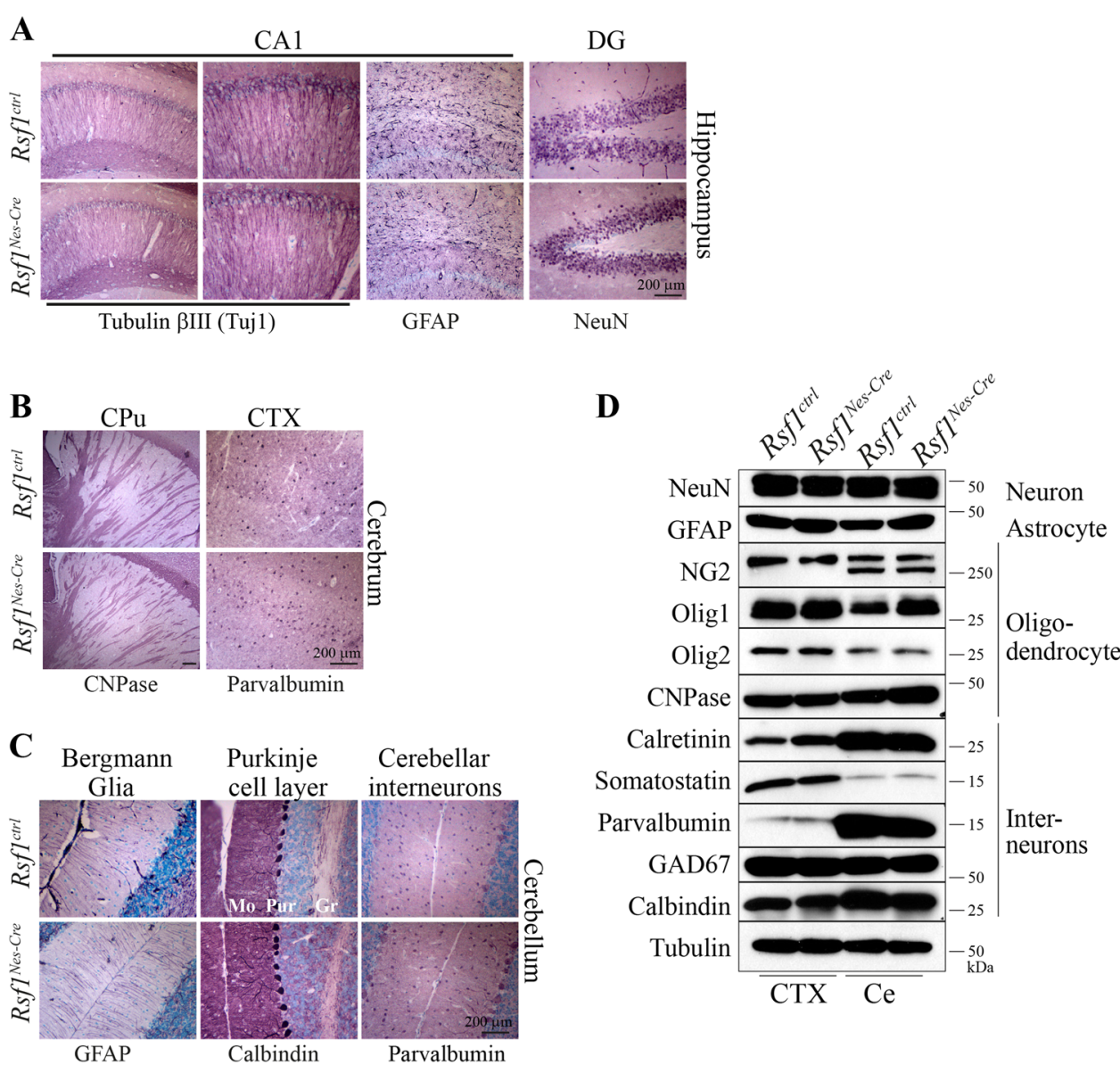

Fig. 1 Rsf1 cKO brain develops normally. a, b. Immunohistochemical analysis of adult brains (6-month old) with markers for neurons (Tuj1 and NeuN) and astrocytes (GFAP) (a) and oligodendrocytes (CNPase) and parvalbumin positive interneurons (b). There were no neuroanatomical differences between $R s f f^{c t r l}$ and $R s f 1^{\text {Nes-Cre }}$ brains with regard to the hippocampus (CA1, cornu ammonis area 1; DG, dentate gyrus), caudate putamen (CPu), or cerebral cortex (CTX). c Immunohistochemical analysis of Bergmann glia (GFAP), the Purkinje cell layer (calbindin), and interneuron/Purkinje cells (parvalbumin) of the mature cerebella (Ce). No difference was found between the Rsf1 ${ }^{\text {ctrl }}$ and Rsf $7^{\text {Nes-Cre }}$ animals. Mo molecular layer; Pur Purkinje cell layer; Gr granule cell layer. d Western blot analysis of the mature (2-month old) brains with markers for neurons (NeuN), astrocytes (GFAP), oligodendrocytes (NG2, Olig1 and 2, and CNPase), and interneurons (calretinin, somatostatin, parvalbumin, GABA[GAD67], and calbindin)

regulates p53 transcriptional activity and its deficiency results in the aberrant transcriptional response of p53target genes upon DNA damage.

Next, the binding of p53 to the target genes, particularly to p53 response elements (p53RE), which is a necessary step to regulate gene expression, was examined in RSF1 KO cells. Chromatin immunoprecipitation (ChIP) was performed with p53 antibodies in RSF1 WT Ctrl and KO cells. The fold enrichment of p53 at p53RE and nonp53RE sites in the CDKN1A (p21) and BBC3 (PUMA) promoter, and p53REs in $B A X$ and PMAIP1 (NOXA) after the drug treatment was assessed in RSF1 WT Ctrl and KO U2OS cells. p53 binding on p53RE in the target genes was significantly enriched after etoposide treatment in U2OS WT Ctrl cells (Fig. 4b). However, p53 binding to p53REs of apoptotic genes (PUMA, BAX, and PMAIP1) was significantly reduced in RSF1 KO cells after DNA strand breaks (Fig. 4c), yet difference of p53 binding to CDKN1A was not statistically significant in this experimental setting. Thus, $R S F 1$ deficiency impairs the p53 activity and its binding to target gene promoters and p53RE, resulting in lower gene-expression levels, particularly related to apoptosis, upon DNA strand breaks induced by etoposide treatment.

p53 forms complexes with the histone acetyltransferase p300 for transcriptional activation, such that p300 can either acetylate histones near the target promoters, thereby relaxing the chromatin structure to promote transcription, or acetylate p53 itself, thus stabilizing the protein to regulate the transcription of p53-target genes ${ }^{30}$. Accordingly, ChIP experiment with p300 antibody showed the enrichment of p300 on both p53RE and nonp53RE in the promoters of p53-target genes after etoposide treatment (Fig. 5a and Figure S3a). Compared to 

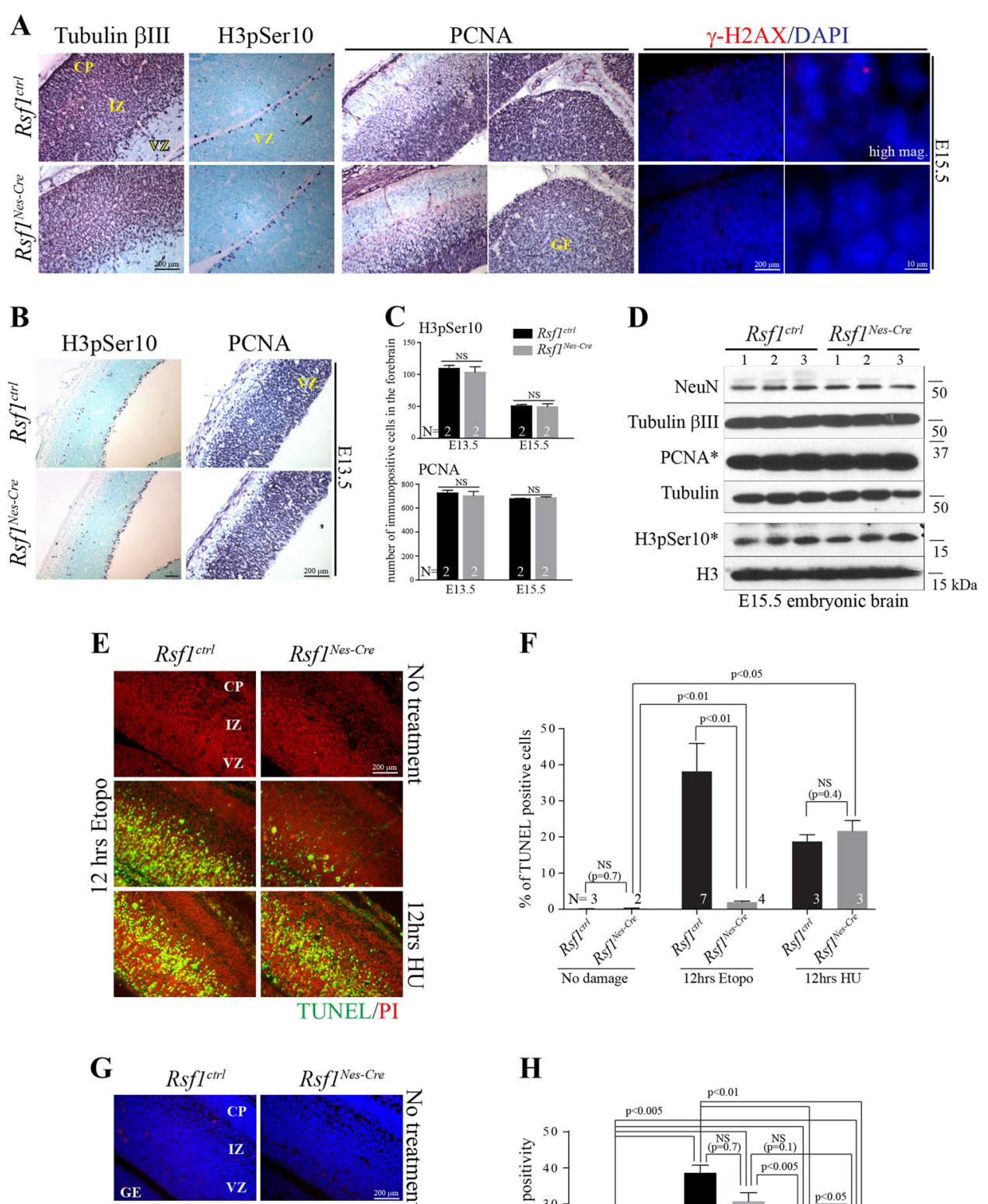

H

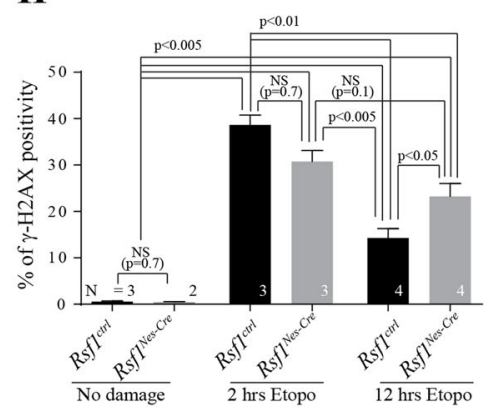

$\gamma-\mathrm{H} 2 \mathrm{AX} / \mathrm{DAPI}$

Fig. 2 (See legend on next page.)

WT cells, p300 association with promoters of p53-target genes was reduced in RSF1 KO cells (Fig. 5b and Figure S3b). Moreover, the acetylation of histone substrates, particularly histone $\mathrm{H} 3$, was increased in $B B C 3$ promoters and enriched with p300/p53 complex after etoposide treatment in WT Ctrl cells. However, its acetylation was reduced in RSF1 KO cells (Fig. 5c, d), indicating that the chromatin structure is not permissive for apoptotic gene expressions in RSF1 deficiency upon DNA strand breaks. We further tested whether $p 300$ deficiency could affect 
(see figure on previous page)

Fig. 2 Rsf1 deficiency prevents neural death from exogenously induced DNA damage during neurogenesis. a Immunohistochemical analysis of embryonic day (E) 15.5 mouse embryos with markers for neuronal differentiation (tubulin BIII), proliferation (PCNA), and neuroprogenitor cells at metaphase (H3pSer10). The overall neural development of Rsfi ${ }^{\text {Nes-Cre }}$ embryos was comparable with that of Rsf1 Ctrl embryonic brain. Nuclear $\gamma_{-}-\mathrm{H} 2 \mathrm{AX}$ foci were not noticeable in both Rsf1 Ctrl and cKO embryonic brains. $\mathrm{Y}-\mathrm{H} 2 \mathrm{AX}$ immunoreactivity is used to detect endogenous DNA damage during neurogenesis. CP cortical plate; IZ intermediate zone; VZ ventricular zone; GE ganglionic eminence; high mag. higher magnification. $\mathbf{b}$ Immunohistochemical analysis of E13.5 mouse embryos with H3pSer10 and PCNA. c Quantification analysis of H3pSer10 and PCNA immunopositive cells in the ventricular zone of the developing forebrain at E13.5 and E15.5. NS not significant; N numbers of embryos analyzed (multiple sections of embryonic brain were obtained.) d Western blot analysis of several Rsf1 Ctrl and cKO embryonic brains at E15.5. *Proliferation markers. NeuN and Tubulin $\beta$ III (neuronal differentiation and maturation), tubulin and Histone 3 were used for loading control. e, f. Examination of neural apoptosis via TUNEL (e) and quantification (f) after DNA damage induced by etoposide (Etopo) and hydroxyurea (HU) (12 h posttreatment) at E15.5. Green signals indicate TUNEL-positive staining with PI (red) counterstaining. A dramatic difference in apoptosis signal was seen in the Rsf $f^{\text {Nes-Cre }}$ embryonic brain after etoposide treatment, yet no difference was found after $\mathrm{HU}$ treatment. $\mathbf{g}, \mathbf{h}$ DNA damage visualized as nuclear foci formation using $\mathrm{Y}$-H2AX immunoreactivity $(\mathbf{g})$ and quantification (h) in the embryonic forebrains at 2 and $12 \mathrm{~h}$ after Etoposide (Etopo) treatment. The strong nuclear staining of $\mathrm{Y}-\mathrm{H} 2 \mathrm{AX}$ found in $\mathrm{Rsfl}^{c t r l}$ embryonic brains at $12 \mathrm{~h}$ time point after drug treatment is false positive. $\mathrm{N}$ numbers of embryos analyzed (multiple sections of embryonic brain were obtained)

this regulation. The expression of $p 300$, which was suppressed using siRNA (Figure S3c), led to dramatic reduction of p53 activity without DNA damage, even though DNA damage-induced p53 activity in this experimental condition (Fig. 4e), suggesting that posttranslational modification of p53 is important for its activity. This upregulation after DNA damage was abolished in RSF1 deficiency (Fig. 4e), implying the critical role of RSF1 in p53 transcriptional activity upon DNA damage. Taken all these together, the results suggest that chromatin modification by RSF1 and p300 acetyltransferase on p53 REs of its target genes is necessary for proper p53 transcriptional activity upon DNA strand breaks.

\section{Discussion}

The structure of chromatin in higher organisms may present a hurdle toward the repair of DNA damage, as access to the sites of damage is crucial. Thus, the regulation of chromatin dynamics by chromatin-remodeling factors is requisite for proper responses to DNA damage ${ }^{2}$. The chromatin-remodeling factor RSF1 is involved in the DDR in vitro, particularly in the ATM-dependent checkpoint signaling pathway, and in DNA doublestrand break repairs ${ }^{15-17}$. Considering the occurrence of endogenous DNA strand breaks during brain development $^{22}$, the results of the current study show that Rsf 1 is dispensable for murine brain development and is not involved in the repair mechanisms for endogenously induced DNA damage during brain development. However, we could not completely rule out the possibility that RSF1 might play an important function in development of other organs. According to the IMPC website (http:// www.mousephenotype.org), a germline deletion of Rsf1 results in preweaning lethality for unknown reasons. It is possible that other binding partners of SNF2h ATPase, such as WSTF (tyrosine-protein kinase, BAZ1B) and ACF1 (bromodomain adjacent to Zinc finger domain 1A, BAZ1A), that play roles in chromatin remodeling and DDR, possibly compensate for Rsf1 deficiency during brain development ${ }^{31,32}$. It was reported that deletion of $S n f 2 h$ in the mouse results in early embryonic lethality and inactivation of $\mathrm{Snf} 2 \mathrm{~h}$ during neurogenesis caused cerebellar ataxia ${ }^{25,33}$. Nevertheless, cells in the brains of Rsf1 cKO mice were not apoptotic after etoposide treatment but were after treatment with $\mathrm{HU}$, similar to a previous demonstration of different sensitivities of RSF1 knockdown cells to DNA double- and single-strand breaks $^{15}$.

The impaired cell death response with RSF1 deficiency following excessive DNA strand breaks was confirmed by in vitro and in vivo analyses, which identified the reduced transcription of p53-target genes as a potential mechanism. The reduction in target gene expression after DNA damage was associated with a loss of p53 or p300 binding at the gene promoters and p53REs. Moreover, the acetylation of histones was reduced at these promoter regions after induction of DDR in RSF1 deficiency. This reduction in binding was not a result of reduced $\mathrm{p} 53$ protein levels, which the protein was unchanged and comparably stabilized after DNA damage in mouse brain tissues and human RSF1 KO cells. However, the posttranslational modification of p53 by p300 as a cause of reduced target gene expression cannot be ruled out, as the acetylation of K120 and C-terminal lysines of p53 is known to regulate the transcription of pro-apoptotic genes ${ }^{30,34}$. Thus, further study of p53 acetylation and phosphorylation in RSF1 $\mathrm{KO}$ cells after DNA damage is warranted. Furthermore, RSF1 might directly mediate the interaction of p53 with p300 to modify the chromatin structure and activate the 

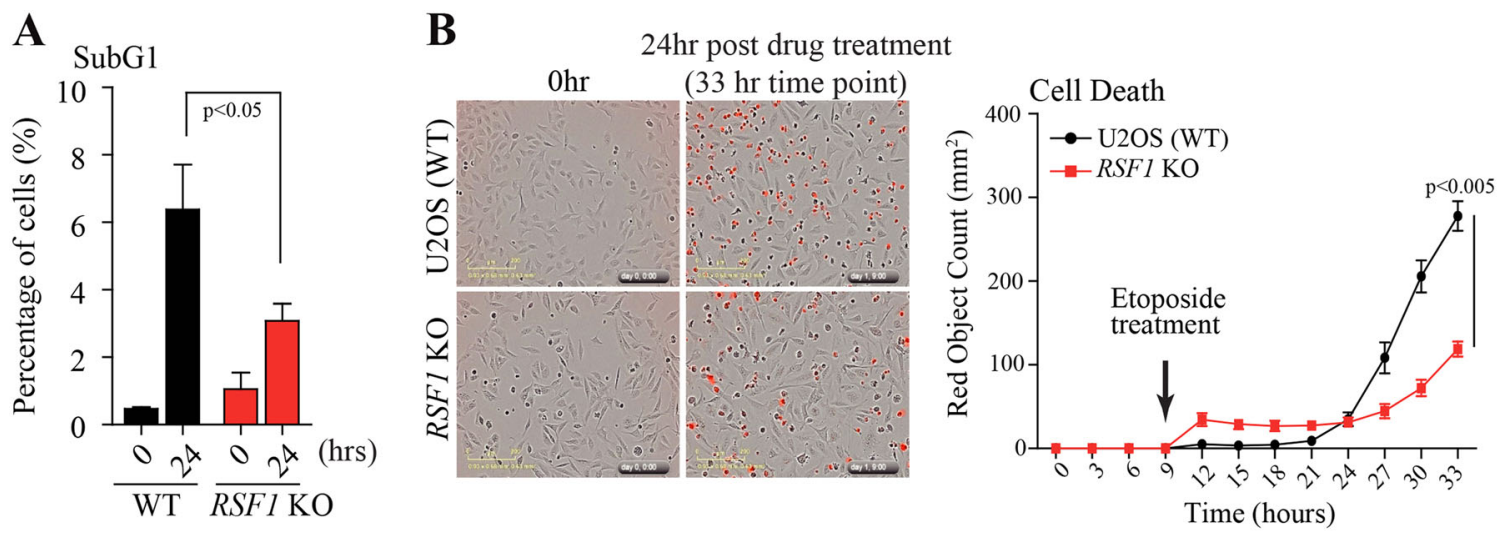

C

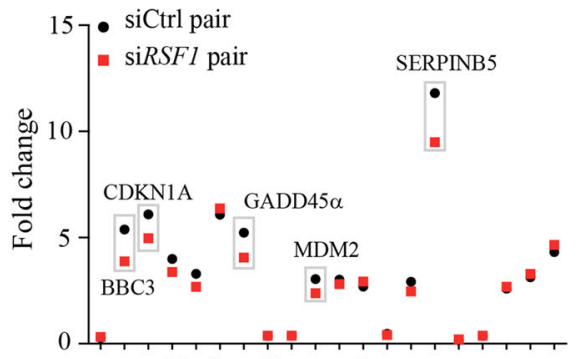

D
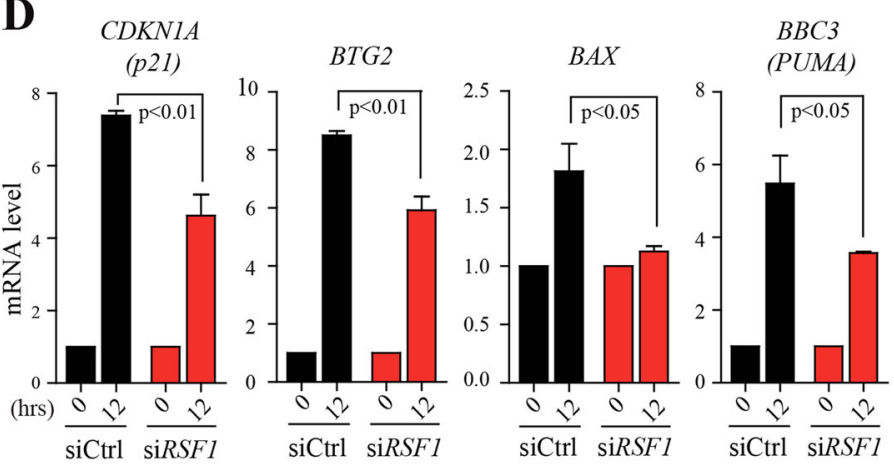

$\mathbf{E}$
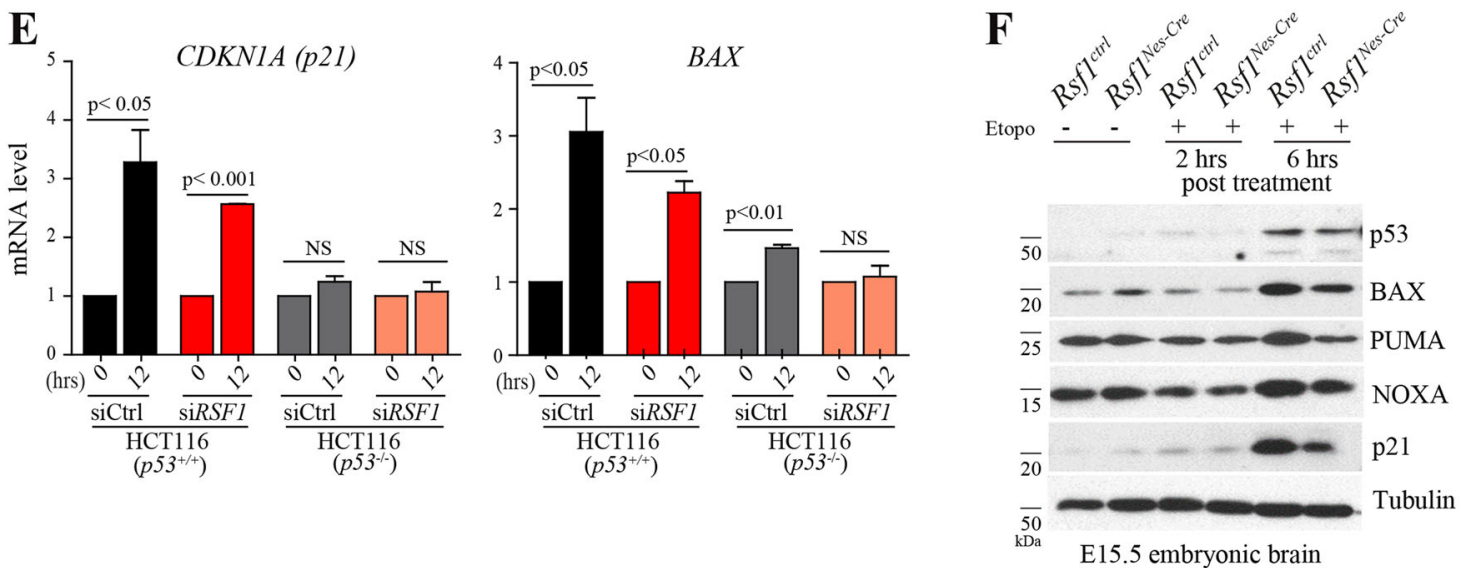

Fig. 3 RSF1 KO hinders p53 signaling to reduce cell death after DNA damage. a SubG 1 populations of U2OS WT and RSF1 KO cells after treatment with etoposide $(68 \mu \mathrm{M})$ for $24 \mathrm{~h}$ stained with PI and counted by FACS. b U2OS wild-type (WT) and RSF1 KO cells were treated with etoposide and IncuCyte Cytotox Red reagent simultaneously. Cell death indicated by red fluorescence was imaged every $3 \mathrm{~h}$ for $24 \mathrm{~h}$ after drug treatment, and counted by IncuCyte. c Scatter plot of fold changes of gene expression in the p53 signaling pathway in RSF1 WT and KO cells. The same gene pair between Ctrl and RSF1 KO cells is marked in a gray box. d Fold induction in the expression of p53-target genes (CDKN1A, BTG2, BAX, and PUMA) in RSF1 WT Ctrl and deficient U2OS cells after etoposide treatment was confirmed by quantitative PCR. Gene expression induced by drug treatment was normalized to the basal level without drug treatment. e Fold induction in the expression of p53-target genes (CDKN1A and BAX) in HCT116 p53 proficient $\left(p 53^{+/+}\right)$and deficient $\left(p 53^{-/-}\right)$cells after etoposide treatment was confirmed by quantitative PCR. siRSF1 was also applied. Gene expression induced by drug treatment was normalized to the basal level without drug treatment. $\mathbf{f}$ Western blot analysis of p53-target proteins (BAX, PUMA, NOXA, and p21) in the embryonic brains at E15.5 after etoposide treatment. The similar level of p53 stabilization in both Rsf1 Ctrl and CKO embryonic brains was detected after etoposide treatment 


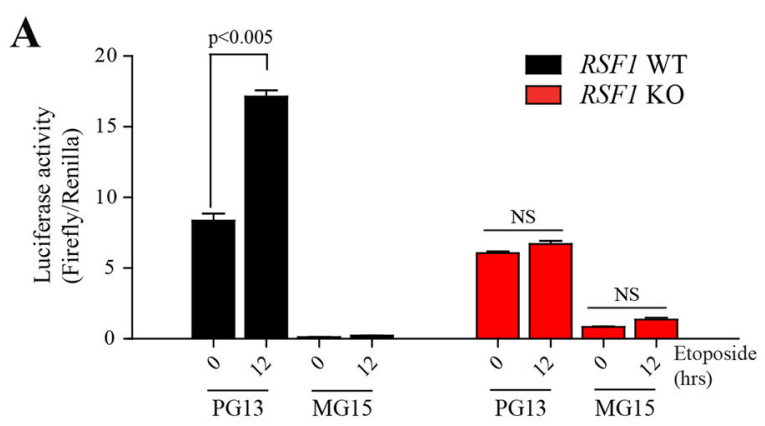

B

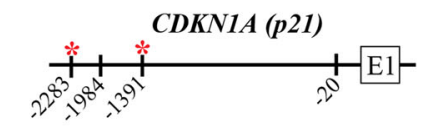

p53 binding in $C D K N 1 A$

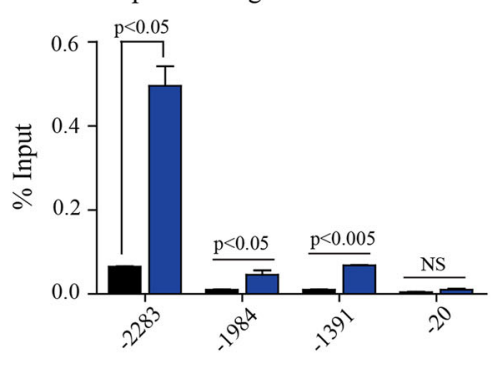

C

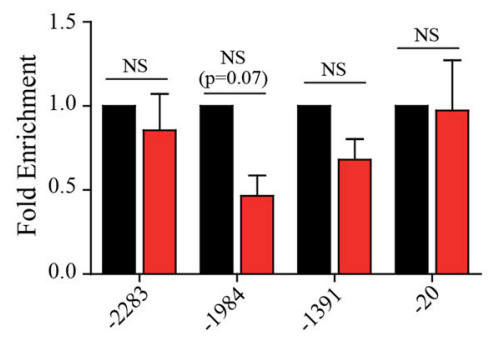

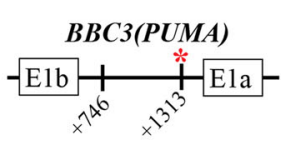

p53 binding in $B B C 3$

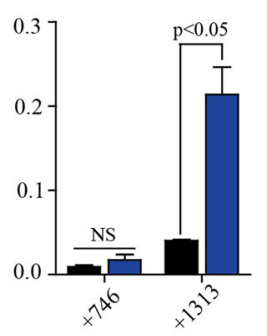

p53 binding in $B B C 3$

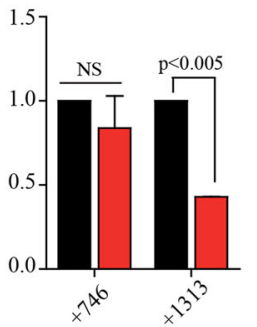

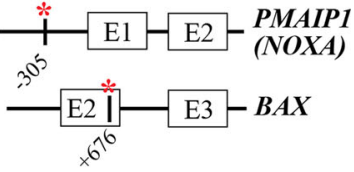

p53 binding in $B A X \& N O X A$

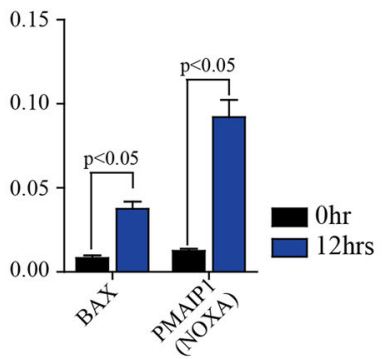

p53 binding in $B A X \& N O X A$

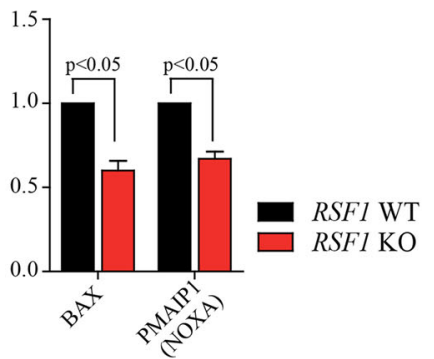

Fig. 4 RSF1 KO regulates binding of p53 to target gene promoters. a Luciferase assay to measure p53 activity for transcriptional regulation upon DNA damage. PG13 contains 13 repeats of p53 binding sites, and MG15 contains 15 repeats of the mutated binding site. NS not significant. $\mathbf{b}$ ChIP analysis of p53 at the promoters of CDKN1A (p21, left, with a schematic diagram of the promoter region of CDKN1A) and BBC3 (PUMA, middle, with a schematic diagram of the promoter region of $B B C 3$ ), and the p53 response elements (RE) of $B A X$, and PMAIP1 (NOXA, right, with schematic diagrams of the p53-RE locations). The enrichment of p53 on these promoters after treatment with etoposide was calculated and represented in \% input. In schematic diagrams of genes, red asterisks indicate p53 response elements in the promoters or the gene body. c Comparison of ChIP analysis of p53 at the promoters of CDKN1A and BBC3, and the p53 response elements (RE) of BAX, and PMAIP1 between RSF1 WT and KO cells. The level of enrichment of p53 at the promoters and p53REs after treatment with etoposide was normalized to the level before treatment in control cells

transcription of the target genes. As the RSF complex was initially purified with the FACT complex and identified as a transcriptional activator, it is also possible that the RSF1/SNF2h complex regulates histone modifications of $\mathrm{H} 3 / \mathrm{H} 4$ and recruitment of p300 to specific promoters ${ }^{3,4}$.

In summary (Fig. 6), the current study shows that a loss of RSF1 impairs apoptosis and p53-dependent gene expression, particularly of genes related to cell death, after etoposide treatment in vivo and in vitro. The reduction in p53-induced gene expression was associated with a loss of p53/p300 binding at the gene promotors accompanied by altered histone modifications, likely resulting in altered chromatin structure and genomic instability. 

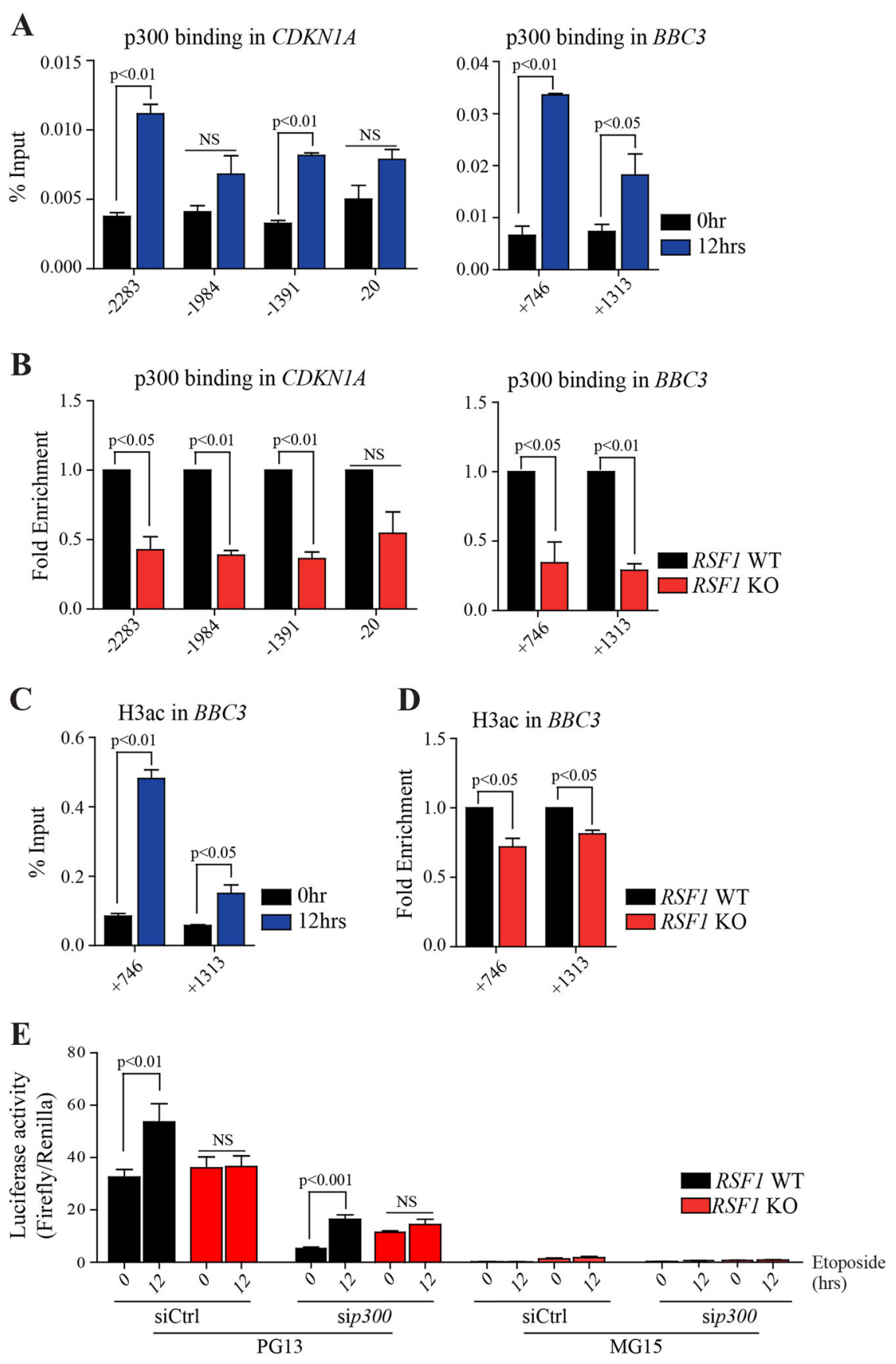

Fig. 5 RSF1 KO impairs p300 binding and the subsequent histone $\mathrm{H} 3$ acetylation on p53-target promoters. a ChIP analysis of histone p300 at the promoters of CDKN1A (p21, numbers: see Fig. 4) and BBC3 (PUMA, middle, numbers: see Fig. 4), and p53 response elements of BAX, and PMAIP1 (NOXA, the locations: see Fig. 4). The enrichment of p300 on these promoters after treatment with etoposide was calculated and represented in \% input. NS not significant. $\mathbf{b}$ The level of enrichment of p300 at the promoters after treatment with etoposide in RSF1 KO cells was normalized to the level before treatment in control cells. NS not significant. c ChIP analysis of histone H3 acetylation (H3ac) at the promoters of BBC3 (PUMA). The enrichment of histone $\mathrm{H} 3$ acetylation on these promoters after treatment with etoposide was calculated and represented in \% input. $\mathbf{d}$ The level of enrichment of histone $\mathrm{H} 3$ acetylation at the promoters after treatment with etoposide in RSF1 KO cells was normalized to the level before treatment in control cells. e Luciferase assay to measure p53 activity for transcriptional regulation upon DNA damage. The activity of p53 for transcription was measured in the condition of $p 300$ or RSF1 deficiency, as well as double deficiency of p300 and RSF1 after etoposide treatment. PG13 contains 13 repeats of p53 binding sites, and MG15 contains 15 repeats of the mutated binding site. NS not significant 


\section{Materials and methods Animals}

The animal model harboring a floxed exon 4 of the Rsf1 gene $\left[R s f 1^{t m 1 c(E U C O M M)}\right]$ after breeding with a Flp animal line to remove the LacZ/Neo cassette flanked by the Frt sites (S1b) was obtained from the Toronto Centre for Phenogenomics (a facility of Mount Sinai Hospital \& the Hospital for Sick Children, Canada), which is a member of the international mouse phenotyping consortium (IMPC). Up to date, the IMPC website (http://www. mousephenotype.org/data/genes/MGI:2682305) indicates that the Rsf1 $1^{\operatorname{tm} 1 b(E U C O M M)}$ allele, which is a reportertagged deletion allele, results in preweaning lethality. To induce Rsf1 inactivation during neurodevelopment, Rsf1 $1^{\text {LoxP/LoxP }}$ animals were crossed with Nestin-Cre [B6. $\mathrm{Cg}-\mathrm{Tg}(\mathrm{Nes}-\mathrm{cre}) 1 \mathrm{Kln} / \mathrm{J}$, stock no. 003771] animals to generate Rsf1 ${ }^{\text {LoxP/LoxP }}$;Nestin-Cre (Rsf1 ${ }^{\text {Nes-Cre }}$ or Rsf1 cKO) animals. There were no differences between male and female Rsf1 cKO mice; thus analyses were performed regardless of sex. The Rsf $1^{\text {LoxP/+ }}$;Nestin-Cre animals did not show any defects and were used as the controls. The genotypes of Rsf1 animals were determined by routine PCR using the following primers. As shown in Figure S1b, to detect the floxed allele, Set1 primers were used [P1: $5^{\prime}$ TTGGAGAGATTTCGGGTAAACTTAC (forward) and P2: 5'-CAATACATGAGGCCATCTTTGTCTC

(reverse)] with 34 cycles of amplification at $94^{\circ} \mathrm{C}$ for $30 \mathrm{~s}$, $60{ }^{\circ} \mathrm{C}$ for $45 \mathrm{~s}$, and $72{ }^{\circ} \mathrm{C}$ for $1 \mathrm{~min}$, which produces a 667 bp fragment for the WT allele and an $808 \mathrm{bp}$ fragment for the floxed allele. To detect the targeted allele, Set 2 primers were used [P3: 5'-GCGCAACGCAATTAAT GATAAC (forward) and P4: 5'-ACCAGGTAA GAGTTCATGTCAAAGCAGC (reverse)] with 34 cycles of amplification at $94{ }^{\circ} \mathrm{C}$ for $30 \mathrm{~s}, 60^{\circ} \mathrm{C}$ for $30 \mathrm{~s}$, and $72{ }^{\circ} \mathrm{C}$ for $1 \mathrm{~min}$, which produces a $437 \mathrm{bp}$ fragment. The PCR for Cre recombinase was detected using the following primers and PCR conditions: (Cre-3: 5'-CTGCCAC GACCAATGACAGC and Cre-4: 5'-ACCTG CGGTGCTAACCAGCG) with 35 cycles of amplification at $94{ }^{\circ} \mathrm{C}$ for $30 \mathrm{~s}, 60^{\circ} \mathrm{C}$ for $45 \mathrm{~s}$, and $72{ }^{\circ} \mathrm{C}$ for $45 \mathrm{~s}$.

The presence of a vaginal plug indicated embryonic day 0.5 (E0.5), and the day of birth was designated as postnatal day 0 (P0). To induce DNA damage, etoposide $(4 \mathrm{mg} / \mathrm{kg}$ ) or $\mathrm{HU}(400 \mathrm{mg} / \mathrm{kg})$ was administrated intraperitoneally to pregnant mice at E15.0, and the embryos were collected at the indicated time points. All animals were housed in the Laboratory Animal Research Center of Ajou University Medical Center and maintained in accordance with the guidelines of the Institutional Animal Care and Use Committee. All procedures for animal use were approved by the ethics committee at the center.

\section{Reverse transcription and quantitative real-time PCR}

To measure effective deletion of floxed exon 4 in the Rsf1 gene, quantitative real-time PCR was applied. Genomic DNA was purified from tissue samples with a phenol/chloroform solution and used for quantitative real-time PCR on a Rotor-Gene Q instrument (Qiagen) with a Rotor-Gene SYBR green PCR kit (Qiagen) and the following primers. The floxed exon 4 of Rsf1 was amplified with primers: 5'-GGTACCAGTTGGATCAA GACCATA (forward) and 5'-ACAATGCATTTCCAA GAAGAGCCA (reverse); the intact exon 6 was amplified with primers: 5'-GGACATGCCTCTCGAACCTT (forward) and 5'-CTTCTGGGGGCCTTCTCTTC (reverse). The results of real-time PCR were normalized to those for $\beta$-actin (forward primer: 5'-CAGCAAGCAGGAGTAC GATGAG; reverse primer: 5'-CAGTAACAGTC CGCCTAGAAGCA). The deletion efficacy by Cre recombinase was calculated as the ratio of exons 4 and 6 .

For quantitative reverse transcription real-time PCR to confirm RNAseq analysis, $2 \mu \mathrm{g}$ of total RNA isolated from several human cell lines as described below was used for cDNA synthesis with amfiRivert cDNA synthesis platinum master mix (GenDEPOT). Quantitative PCR was performed on a Rotor-Gene Q system (Qiagen) using Maxima SYBR Green qPCR master mix (Thermo) with the indicated primers (Table S1).

\section{Cloning of mouse Rsf1 for microirradiation}

The cloning of human RSF1 was described previously $^{15}$. Murine Rsf1 was amplified from mouse brain CDNA via routine PCR, and the PCR product was cloned into a GFP-tagged vector using the Gateway cloning system (Invitrogen). For DNA damage analysis, U2OS cells were transfected with either human RSF1-GFP or mouse Rsf1-GFP. When the cells reached $\sim 80 \%$ confluency, BrdU (final concentration, $10 \mu \mathrm{M}$ ) was added to the culture for $30 \mathrm{~h}$ prior to microirradiation, which was conducted with a $405 \mathrm{~nm}$ laser (32 repetitive insults/s for $3 \mathrm{~s}$ ) to induce DNA strand breaks. An accumulation of GFP fluorescence at the sites of DNA damage was observed under an A1 confocal microscope (Nikon) equipped with a temperature- and $\mathrm{CO}_{2}$-controlled chamber.

\section{Cell lines and luciferase assay}

For in vitro study, human cell lines [HCT116 $\left(p 53^{+/+}\right)$, HCT116 ( $\left.p 53^{-/-}\right)$, U2OS] RSF1 proficient and deficient cell line were used for the analysis. siRNA sequence for p300 was 5'-AACCCCUCCUCUUCAGCACCA. siRSF1 was previously reported ${ }^{15}$. Luciferase assays were performed in human cell lines using PG13-luc, containing 13 

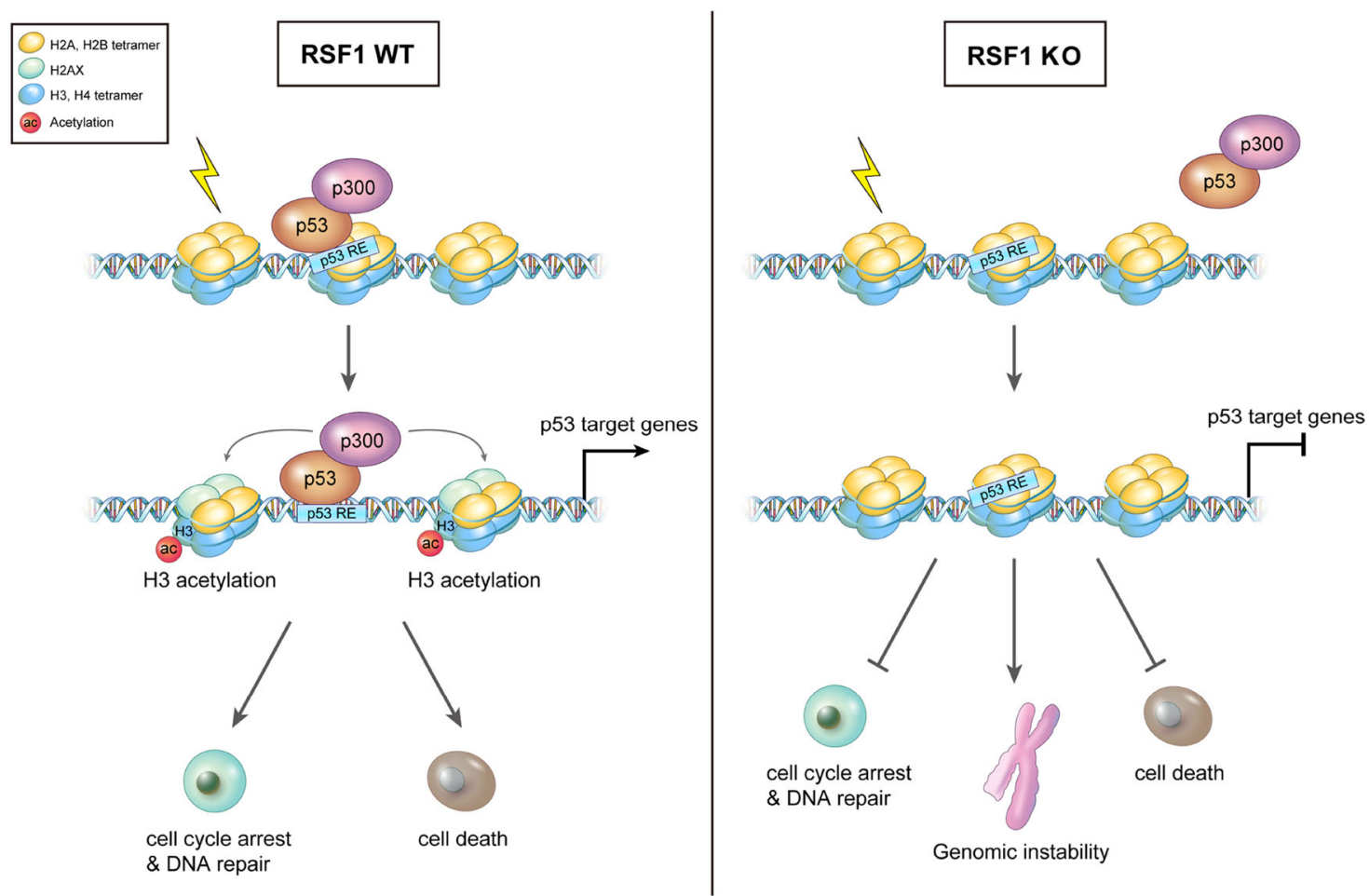

Fig. 6 A graphic summary of the findings. In RSF1 proficiency, p53/p300 complex stably associates with promoters (p53 RE; reponse element) of p53-target genes, which leads to the subsequent histone $\mathrm{H} 3$ acetylation on the promoters to induce gene expression upon DNA damage. However, RSF1 inactivation blocks the stable association of p53/p300 complex on the promoters of p53-target genes and suppresses their expressions, especially apoptotic genes. Consequently, this condition leads to escape from cell death, resulting in genomic instability

copies of the p53 binding consensus sequence (PG13), and MG15-luc, containing 15 copies of mutated p53 binding sequence (MG15), reporter plasmids (Addgene). Cells were transfected with the reporter plasmids and siRNA using Lipofectamine 2000 (Invitrogen) and incubated for $48 \mathrm{~h}$. Etoposide was treated for $12 \mathrm{~h}$ before cells were harvested. Luciferase activity was measured using a Dual-Luciferase Reporter Assay System (Promega). Firefly luciferase activity was normalized by Renilla luciferase activity, and statistically analyzed using Prism software (GraphPad).

\section{Western blot analysis}

Mature and embryonic brain samples were microdissected from 2 to 3 Rsf 1 Ctrl or cKO animals and snapfrozen in liquid nitrogen. Western blotting was performed as described before with a slight modification ${ }^{15}$. Mouse brain tissues, RSF1 proficient and deficient human cell lines were prepared in a lysis buffer containing $50 \mathrm{mM}$ Tris (pH 7.5), $150 \mathrm{mM} \mathrm{NaCl}, 50 \mathrm{mM} \mathrm{NaF}, 0.2 \% \mathrm{NP}-40,1 \%$ Tween-20, $1 \mathrm{mM}$ dithiothreitol, protease inhibitor (Sigma), and phosphatase inhibitor (Sigma). Protein amounts were quantified using Bio-Rad protein assay reagent. The antibodies for Western blot analyses were against NeuN (1:1000, mouse; Millipore), Tubulin BIII (Tuj1, 1:3000, mouse, BabCo), glial fibrillary acidic protein [(GFAP) 1:2000, rabbit; Cell Signaling Technology, NG2 (1:500, rabbit; Millipore), Olig1 (1:1000, rabbit; LifeSpan Biosciences), Olig2 (1:1000, mouse; Millipore), cyclicnucleotide phosphodiesterase (CNPase) 1:2000, mouse; Sigma, calretinin (1:10,000, rabbit; Millipore), somatostatin (1:1000, rabbit; Abcam), parvalbumin (1:2500, rabbit; Abcam), glutamate decarboxylase] [(GAD67) 1:5000, rabbit; OriGene, calbindin (1:6000, mouse; Sigma)], proliferating cell nuclear antigen [(PCNA) 1:1000, mouse; Santa Cruz Biotechnology], phosphorylated histone 3 serine 10 (H3pSer10, 1:1000, rabbit, Cell signaling), histone 3 (H3, 1:5000, rabbit, Abcam), RSF1 (1:2000, rabbit; Abcam), p53 (1:1000, mouse; Santa Cruz Biotechnology and 1:1000, rabbit, Cell signaling), p21 (CDKN1A, 1:1000, rabbit, Santa Cruz Biotechnology and 1:1000, rabbit, Abcam), BBC3 (Puma, 1:2000, rabbit, Abcam), BAX (1:2000, rabbit, Abcam), Noxa (Pmaip1, 1:2000, mouse, Abcam), p300 (1:1000, Rabbit, Santa Cruz Biotechnology) and GAPDH (1:2000, rabbit; Santa Cruz Biotechnology). The equal loading of samples was confirmed by 
quantifying bands for anti-tubulin (1:40,000, mouse; a gift from Dr. Sang Gyu Park) and Ponceau staining.

\section{Histopathological analysis}

Histopathological analyses were performed as described below. Briefly, embryos and adult mouse brain were fixed in $4 \%$ formaldehyde in phosphate-buffered saline (PBS), cryoprotected in $30 \%$ sucrose PBS solution, and cryosectioned $(10 \mu \mathrm{m}$ thick sagittal sections) with an MEV cryostat (SLEE Medical GmbH, Mainz, Germany). Sections were incubated with antibodies overnight after quenching endogenous peroxidase with $0.6 \%$ hydrogen peroxide for colorimetric signals. Immunopositivity was visualized with the VIP substrate kit (Vector Laboratories) with biotinylated secondary antibodies and avidin DHbiotinylated horseradish peroxidase- $\mathrm{H}$ complex (Vector Laboratories) or with Cy3-conjugated secondary antibodies (Jackson ImmunoResearch Laboratories). Methyl green $(0.1 \%$ solution) was used for counterstaining followed by mounting with DPX (Sigma) or DAPI/propidium iodide (PI) containing mounting medium (Vector Laboratories). Citric acid-based antigen retrieval was applied when necessary. The antibodies were against tubulin BIII (Tuj1, 1:1000, mouse; BabCo), GFAP (1:500, mouse; Sigma), NeuN (1:500, mouse, Millipore), CNPase (1:2000, mouse; Sigma), calbindin (1:2000, mouse; Sigma), parvalbumin (1:1000, rabbit; Abcam), proliferating cell nuclear antigen [(PCNA) 1:500, mouse; Santa Cruz Biotechnology], phosphorylated histone 3 serine 10 (H3pSer10, 1:5000, rabbit, Cell signaling) and $\gamma$-H2AX (1:200, rabbit; Cell Signaling Technology). Apoptosis was measured with an Apoptag fluorescein in situ kit (Millipore).

\section{RNA isolation and RNAseq}

Total RNA was isolated from indicated human cell lines with an RNeasy Plus Mini kit (Qiagen). For the RNAseq analysis, the quality of RNA was checked using Bioanalyzer RNA ChIP (Agilent Technologies). An mRNA library was prepared with a Truseq stranded mRNA kit (Illumina), and RNAseq was performed using NextSeq 500 (Illumina). To analyze RNA sequences, raw reads were preprocessed by base trimming and mapped on the reference sequence, STAR. The counted reads were quantified and analyzed by differentially expressed gene analysis and functional annotation was analyzed by DAVID and IPA. To compare the level of transcripts in control and RSF1 depleted cells, the transcription level after DNA damage was normalized by the transcription level before DNA damage, which represents the fold change of transcripts in each indicated siRNA.

\section{Cell-cycle analysis}

U2OS RSF1 WT and KO cells were harvested after treatment with etoposide $(60 \mu \mathrm{M})$ for $24 \mathrm{~h}$ and fixed with $80 \%$ ethanol overnight at $20^{\circ} \mathrm{C}$. The fixed samples were washed with PBS three times and stained with PI for analysis by FACS.

\section{Chromatin immunoprecipitation (ChIP)}

Indicated human cells were harvested after treatment with etoposide $(30 \mu \mathrm{M})$ for $12 \mathrm{~h}$ and cross-linked with $1 \%$ formaldehyde for $20 \mathrm{~min}$. Cells were lysed with SDS lysis buffer [1\% SDS, $10 \mathrm{mM}$ EDTA, $50 \mathrm{mM}$ Tris (pH 8.1)] supplemented with protease and phosphatase inhibitor cocktail (Thermo) for $10 \mathrm{~min}$ on ice. Cell lysates were sonicated with a Bioruptor (Diagenode) and centrifuged at 13,000 rpm for $15 \mathrm{~min}$. The supernatants were collected and diluted for overnight incubation with primary antibody. Then, $20 \mu$ of protein A agarose/salmon sperm DNA (Millipore) was added to each sample and incubated with rotation at $4{ }^{\circ} \mathrm{C}$. After a $1 \mathrm{~h}$ incubation, the beads were washed with low-salt immune complex wash buffer [0.1\% SDS, 1\% Triton X-100, $2 \mathrm{mM}$ EDTA, $20 \mathrm{mM}$ Tris$\mathrm{HCl}$ (pH 8.1), $150 \mathrm{mM} \mathrm{NaCl}$, high-salt immune complex wash buffer [0.1\% SDS, 1\% Triton X-100, 2 mM EDTA, $20 \mathrm{mM}$ Tris- $\mathrm{HCl}$ (pH 8.1), $500 \mathrm{mM} \mathrm{NaCl}$, LiCl immune complex wash buffer [0.25 M LiCl, 1\% IGEPAL-CA630, $1 \%$ deoxycholic acid (sodium salt), $1 \mathrm{mM}$ EDTA, $10 \mathrm{mM}$ Tris ( $\mathrm{pH}$ 8.1)], and TE buffer $(10 \mathrm{mM}$ Tris- $\mathrm{HCl}, 1 \mathrm{mM}$ EDTA, pH 8.0). The immune complexes were eluted in elution buffer (1\% SDS, $0.1 \mathrm{M} \mathrm{NaHCO}$ ) for $30 \mathrm{~min}$ at room temperature. The eluates were incubated with $10 \mu \mathrm{l}$ of $5 \mathrm{M} \mathrm{NaCl}$ to reverse histone-DNA cross-links by heating at $65^{\circ} \mathrm{C}$ for $4 \mathrm{~h}$, followed by incubation with proteinase $\mathrm{K}$ at $45^{\circ} \mathrm{C}$ for $1 \mathrm{~h}$. DNA was purified with a Nucleospin PCR clean-up kit (Macherey-Nagel) and processed for quantitative PCR. The antibodies used in ChIP assays were against p53 (Santa Cruz Biotechnology), p300 (Santa Cruz Biotechnology), and acetylated H3 (Millipore). The primers used for this analysis are described in Table $\mathrm{S}^{35,36}$.

\section{Image and statistical analyses}

Images from a B600TiFL microscope (Optika) were captured with a DFC130 digital camera (Leica), processed using Photoshop software (Adobe), and analyzed with the measuring function in Image $(\mathrm{NIH})$. Areas of TUNELpositive green signals $\left(0.27 \mathrm{~mm}^{2}\right)$ in the developing forebrains were measured for green and red signals, and then the ratio of the apoptosis signal and total area was calculated. Also H3pSer10 and PCNA immunopositive cells $\left(0.27 \mathrm{~mm}^{2}\right)$ were counted in multiple sections of 
embryonic brains. For DNA damage, the ratio of the $\gamma$ H2AX foci area (red in $150 \mu \mathrm{m}^{2}$ ) and the total nucleus area (blue) was calculated. Multiple slides (apoptosis) and several sites in each section in multiple slides $(\gamma-\mathrm{H} 2 \mathrm{AX})$ were measured, and the number of examined embryos was as indicated in Fig. 2. For cell death analysis in vitro, images from IncuCyte were captured every $3 \mathrm{~h}$ for $24 \mathrm{~h}$ after drug treatment at $9 \mathrm{~h}$ time point (Fig. 3b) and IncuCyte Cytotox Red reagent was automatically counted by IncuCyte. U2OS WT and RSF1 KO cells were treated with IncuCyte Cytotox Red reagent and etoposide simultaneously. All statistical analyses were performed using Prism software (GraphPad). A $P$ value of $<0.05$ was considered significant.

\section{Acknowledgments}

We thank the Laboratory Animal Research Center of Ajou University Medical Center, Korea, for animal husbandry. We also thank Se-Jeong Kim for technical support. Y.L. was supported by a NRF grant funded by the Korea government (MSIP) (2014R1A1A2056224 and 2017R1A2B2009284). This work was also supported by NRF grant (2011-0030043).

\section{Author details}

${ }^{1}$ Genomic Instability Research Center, Ajou University School of Medicine, Suwon 16499, Korea. ${ }^{2}$ Department of Biochemistry \& Molecular Biology, Ajou University School of Medicine, Suwon 16499, Korea. ${ }^{3}$ Department of Biomedical Sciences, The Graduate School of Ajou University, Suwon 16499, Korea. ${ }^{4}$ Genome Stability Institute, Ajou University School of Medicine, Suwon 16499, Korea

\section{Author contributions}

H.C. and Y.L. conceived the scientific idea. S.M., K.K., S.K., and Y.L. performed the experiments. All authors analyzed the data and contributed to writing the manuscript. H.C. and Y.L. finalized the manuscript.

\section{Conflict of interest}

The authors declare that they have no conflict of interests.

\section{Publisher's note}

Springer Nature remains neutral with regard to jurisdictional claims in published maps and institutional affiliations.

Supplementary Information accompanies this paper at (https://doi.org/ 10.1038/s41419-018-1128-2).

Received: 31 July 2018 Revised: 2 October 2018 Accepted: 8 October 2018 Published online: 22 October 2018

\section{References}

1. McKinnon, P. J. DNA repair deficiency and neurological disease. Nat. Rev. Neurosci. 10, 100-112 (2009).

2. Price, B. D. \& D'Andrea, A. D. Chromatin remodeling at DNA double-strand breaks. Cell 152, 1344-1354 (2013).

3. Loyola, A. et al. Functional analysis of the subunits of the chromatin assembly factor RSF. Mol. Cell Biol. 23, 6759-6768 (2003).

4. Loyola, A. \& Reinberg, D. Histone deposition and chromatin assembly by RSF. Methods 31, 96-103 (2003).

5. Brown, L. A. et al. Amplification of 11q13 in ovarian carcinoma. Genes Chromosomes Cancer 47, 481-489 (2008).

6. Chen, T. J., Huang, S. C., Huang, H. Y., Wei, Y. C. \& Li, C. F. Rsf-1/HBXAP overexpression is associated with disease-specific survival of patients with gallbladder carcinoma. APMIS 119, 808-814 (2011).
7. Li, H. et al. Rsf-1 overexpression in human prostate cancer, implication as a prognostic marker. Tumour Biol. 35, 5771-5776 (2014).

8. Liu, S., Dong, Q. \& Wang, E. Rsf-1 overexpression correlates with poor prognosis and cell proliferation in colon cancer. Tumour Biol. 33, 1485-1491 (2012).

9. Maeda, D. et al. Rsf-1 (HBXAP) expression is associated with advanced stage and lymph node metastasis in ovarian clear cell carcinoma. Int J. Gynecol. Pathol. 30, 30-35 (2011)

10. Mao, T. L. et al. Expression of Rsf-1, a chromatin-remodeling gene, in ovarian and breast carcinoma. Hum. Pathol. 37, 1169-1175 (2006).

11. Sheu, J. J. et al. Rsf-1, a chromatin remodelling protein, interacts with cyclin E1 and promotes tumour development. J. Pathol. 229, 559-568 (2013).

12. Shih, le,M. et al. Amplification of a chromatin remodeling gene, Rsf-1/HBXAP, in ovarian carcinoma. Proc. Natl Acad. Sci. USA 102, 14004-14009 (2005).

13. Xie, C., Fu, L., Xie, L., Liu, N. \& Li, Q. Rsf-1 overexpression serves as a prognostic marker in human hepatocellular carcinoma. Tumour Biol. 35, 7595-7601 (2014).

14. Ren, J. et al. Overexpression of Rsf-1 correlates with pathological type, p53 status and survival in primary breast cancer. Int J. Clin. Exp. Pathol. 7, 5595-5608 (2014)

15. min, S. et al. ATM-dependent chromatin remodeler Rsf-1 facilitates DNA damage checkpoints and homologous recombination repair. Cell Cycle 13, 666-677 (2014).

16. Helfricht, A. et al. Remodeling and spacing factor 1 (RSF1) deposits centromere proteins at DNA double-strand breaks to promote non-homologous endjoining. Cell Cycle 12, 3070-3082 (2013).

17. Pessina, F. \& Lowndes, N. F. The RSF1 histone-remodelling factor facilitates DNA double-strand break repair by recruiting centromeric and Fanconi Anaemia proteins. PLoS Biol. 12, e1001856 (2014).

18. McKinnon, P. J. ATM and the molecular pathogenesis of ataxia telangiectasia. Annu Rev. Pathol. 7, 303-321 (2012).

19. Marechal, A. \& Zou, L. DNA damage sensing by the ATM and ATR kinases. Cold Spring Harb. Perspect. Biol. 5, https://doi.org/10.1101/cshperspect.a012716 (2013).

20. Kruse, J. P. \& Gu, W. Modes of p53 regulation. Cell 137, 609-622 (2009).

21. Hanel, W. \& Moll, U. M. Links between mutant p53 and genomic instability. J. Cell Biochem 113, 433-439 (2012).

22. Lee, Y., Choi, I., Kim, J. \& Kim, K. DNA damage to human genetic disorders with neurodevelopmental defects. J. Genet. Med. 13, 1-13 (2016).

23. Sokpor, G., Castro-Hernandez, R., Rosenbusch, J., Staiger, J. F. \& Tuoc, T. ATPdependent chromatin remodeling during cortical neurogenesis. Front Neurosci. 12, 226 (2018).

24. Goodwin, L. R. \& Picketts, D. J. The role of ISWI chromatin remodeling complexes in brain development and neurodevelopmental disorders. Mol. Cell Neurosci. 87, 55-64 (2018).

25. Alvarez-Saavedra, M. et al. Snf2h-mediated chromatin organization and histone $\mathrm{H} 1$ dynamics govern cerebellar morphogenesis and neural maturation. Nat. Commun. 5, 4181 (2014).

26. Lee, H. S. et al. The chromatin remodeller RSF1 is essential for PLK1 deposition and function at mitotic kinetochores. Nat. Commun. 6, 7904 (2015).

27. Barkovich, A. J., Guerrini, R., Kuzniecky, R. I., Jackson, G. D. \& Dobyns, W. B. A developmental and genetic classification for malformations of cortical development: update 2012. Brain 135, 1348-1369 (2012).

28. Lee, Y. \& McKinnon, P. J. ATM dependent apoptosis in the nervous system. Apoptosis 5, 523-529 (2000)

29. Rashi-Elkeles, S. et al. Parallel profiling of the transcriptome, cistrome, and epigenome in the cellular response to ionizing radiation. Sci. Signal 7, rs3 (2014).

30. Reed, S. M. \& Quelle, D. E. p53 Acetylation: regulation and consequences. Cancers (Basel) 7, (30-69 (2014)

31. Xiao, A. et al. WSTF regulates the H2A.X DNA damage response via a novel tyrosine kinase activity. Nature 457, 57-62 (2009).

32. Lan, L. et al. The ACF1 complex is required for DNA double-strand break repair in human cells. Mol. Cell 40, 976-987 (2010).

33. Stopka, T. \& Skoultchi, A. I. The ISWI ATPase Snf2h is required for early mouse development. Proc. Natl Acad. Sci. USA 100, 14097-14102 (2003).

34. Brochier, C. et al. Specific acetylation of p53 by HDAC inhibition prevents DNA damage-induced apoptosis in neurons. J. Neurosci. 33, 8621-8632 (2013).

35. Gomes, N. P. \& Espinosa, J. M. Gene-specific repression of the p53 target gene PUMA via intragenic CTCF-Cohesin binding. Genes Dev. 24, 1022-1034 (2010).

36. Schlereth, $\mathrm{K}$. et al. DNA binding cooperativity of p53 modulates the decision between cell-cycle arrest and apoptosis. Mol. Cell 38, 356-368 (2010). 